\title{
Das Mammakarzinom - Wo stehen wir heute, wo gehen wir hin?
}

$\mathrm{D}$

as Mammakarzinom ist mit über 1 Mio. Neuerkrankungen pro Jahr die weltweit häufigste Tumorerkrankung der Frau. Trotz steigender Inzidenz sinkt die Mortalität. Gründe sind eine verbesserte Diagnostik (Bildgebung und Pathologie) sowie eine standardisierte lokale (Operation und Bestrahlung) und systemische Behandlung. Was sind aber die derzeitigen Standards in der Diagnostik und multimodalen Therapie des Mammakarzinoms? Welche innovativen Entwicklungen erwarten uns in naher Zukunft? Diese Fragen werden im vorliegenden Heft beleuchtet und kritisch diskutiert.

Ein strukturiertes Mammographiescreening senkt die Brustkrebsmortalität um 35\%. Junkermann gibt eine Übersicht zur Leistungsfähigkeit der qualitätsgesichert durchgeführten Mammographie sowie zum derzeitigen Stellenwert von Sonographie und Kernspintomographie. Sinn stellt schlüssig dar, dass das Management der immer häufiger diagnostizierten Vorläuferläsionen nur auf einer modernen, molekularen Pathologie basieren kann. Eine umfassendere Diagnostik genetischer und epigenetischer Veränderungen könnte $\mathrm{zu}$ einer weiter verfeinerten, klinisch wertvollen Reklassifizierung dieser Läsionen führen und damit zu deren adäquaterer Therapie beitragen.

Bei malignen Veränderungen muss der Primärtumor komplett entfernt werden, bei invasiven Anteilen erfolgt auch eine operative Behandlung der axillären Lymphabflusswege. Heutzutage ist meist eine brusterhaltende Operation mit einer weniger radikalen Lymphonodektomie wie der Sentinel-Lymphonodektomie möglich. Wie Rom et al. zeigen, führen moderne, modifizierte Operationstechniken zu weniger kosmetischen und körperlichen Einschränkungen, ohne die Heilungschancen zu verschlechtern. Eine brusterhaltende Operation erfordert eine adjuvante Strahlentherapie. Bei hohem Lokalrezidivrisiko ist eine Strahlentherapie auch nach Mastektomie indiziert. Hof gibt einen umfassenden Einblick in Standards und neue Bestrahlungstechniken wie die intensitätsmodulierte Radiotherapie (IMRT), durch die auch in anatomisch ungünstigen Situationen eine maximale Normalgewebsschonung erreicht werden kann. Möglichkeiten und Grenzen der Teilbrustbestrahlung und die Schwierigkeiten bei der Indikationsstellung zur Bestrahlung der Lymphabflusswege werden kritisch diskutiert.

Ebenso wie die lokalen Therapiemaßnahmen haben Hormon- und Chemotherapie beim primären Mammakarzinom das rezidivfreie Überleben und das Gesamtüberleben signifikant verbessert. Spätestens beim Lesen der umfassenden Übersicht von Aigner \& Schneeweiss wird klar, dass neue zielgerichtete Therapien (Antikörper, klei- ne Moleküle) und Bisphosphonate unsere therapeutischen Optionen zwar stark erweitern, aber auch differenzierter und komplexer werden lassen. In den kommenden Jahren werden weitere Optionen hinzukommen. Experimentelle Ansätze wie die zelluläre Immuntherapie könnten eine klinische Bedeutung erlangen. Domschke et al. geben in ihrem Beitrag einen umfassenden Einblick in die langwierige präklinische Entwicklung dieser Ansätze, beschreiben aber auch erste vielversprechende Ergebnisse einer adoptiven Immuntherapie mit ex vivo stimulierten tumorspezifischen Gedächtnis-T-Zellen.

Die wichtigste Aufgabe der kommenden Jahre wird sicher die schnelle Translation dieser neuen Techniken und Therapieoptionen in das multimodale Behandlungskonzept des Mammakarzinoms sein. Dies kann nur durch qualitativ hochwertige Studien mit innovativen translationalen Begleitprogrammen erreicht werden. Gleichzeitig gilt es, das Ausmaß der Über-, Unter- und Fehlbehandlung durch die Entwicklung neuer Biomarker zu reduzieren. Moderne Hochdurchsatzverfahren erlauben uns schon heute, die molekulare Vielfalt des Mammakarzinoms (und der betroffenen Patientinnen) besser abzubilden als je zuvor. Ihre Möglichkeiten sind enorm. Es gilt, sie rasch zu entwickeln.

Andreas Schneeweiss 